\title{
Quantum Complexity: Some Recent Results, Some Open Problems, Some Thoughts
}

\author{
Richard J. Lipton \\ College of Computing \\ Georgia Institute of Technology \\ Atlanta, GA 30332, USA \\ rjlecc.gatech.edu
}

\begin{abstract}
This talk will discuss some recent results on the complexity theory of quantum computing. The long term goal is to understand exactly the relationship between the new quantum complexity classes and the classic ones. It is assumed that you are not an expert in: quantum flavordynamics, quantum geometrodynamics, quantum hydrodynamics, quantum magnetodynamics, quantum triviality, Schrödinger's equation, path integral, Schwinger-Dyson equation, static forces and virtual-particle exchange, and the Ward-Takahashi identity.

It is assumed that you are interested in hearing some simple ideas that connect basic complexity theory with quantum computation. No previous knowledge is assumed, all will be explained, at least that is the plan of the talk.
\end{abstract}

